\title{
How to inhibit a distractor location? Statistical learning versus active, top-down suppression
}

\author{
Benchi Wang ${ }^{1} \cdot J^{\prime}$ Theeuwes ${ }^{1}$
}

Published online: 23 February 2018

(C) The Psychonomic Society, Inc. 2018

\begin{abstract}
Recently, Wang and Theeuwes (Journal of Experimental Psychology: Human Perception and Performance, 44(1), 13-17, 2018a) demonstrated the role of lingering selection biases in an additional singleton search task in which the distractor singleton appeared much more often in one location than in all other locations. For this location, there was less capture and selection efficiency was reduced. It was argued that statistical learning induces plasticity within the spatial priority map such that particular locations that are high likely to contain a distractor are suppressed relative to all other locations. The current study replicated these findings regarding statistical learning (Experiment 1) and investigated whether similar effects can be obtained by cueing the distractor location in a top-down way on a trial-by-trial basis. The results show that top-down cueing of the distractor location with long (1,500 ms; Experiment 2) and short stimulus-onset symmetries (SOAs) (600 ms; Experiment 3) does not result in suppression: The amount of capture nor the efficiency of selection was affected by the cue. If anything, we found an attentional benefit (instead of the suppression) for the short SOA. We argue that through statistical learning, weights within the attentional priority map are changed such that one location containing a salient distractor is suppressed relative to all other locations. Our cueing experiments show that this effect cannot be accomplished by active, top-down suppression. Consequences for recent theories of distractor suppression are discussed.
\end{abstract}

Keywords Attentional capture $\cdot$ Suppression $\cdot$ Statistical learning $\cdot$ Top-down $\cdot$ Cueing

\section{Introduction}

The visual world we encounter in everyday life is complex and filled with an enormous amount of perceptual information. In order to act coherently, we have to focus our limited resources on relevant information and filter out distracting information. Selective attention is the mechanism that determines what we see and act upon. Traditionally, it has been assumed that attentional selection is controlled by the interaction between top-down and bottom-up processes, with the former determined by current selection goals and the latter determined by physical salience. Recently, it was recognized that many instances of attentional selection are not the result of

Benchi Wang

wangbenchi.swift@gmail.com

1 Department of Experimental and Applied Psychology, and Institute Brain and Behavior Amsterdam (iBBA), Vrije Universiteit

Amsterdam, Van der Boechorststraat 1, 1081

BT Amsterdam, The Netherlands top-down nor of bottom-up processing, but are instead due to the "history" of former attention deployment that can elicit lingering selection biases that go beyond goal-based or salience-based selection (Awh, Belopolsky, \& Theeuwes, 2012; see also Anderson, 2016; Jiang, 2017).

Recently, Wang and Theeuwes (2018) demonstrated the role of lingering selection biases in an additional singleton search task in which the distractor singleton appeared much more often in one location than in all other locations. The results showed that a distractor singleton in the highprobability location interfered much less with searching for the target than when the same distractor singleton appeared in another location. Also, when the target singleton happened to appear in that high-probability distractor location, its selection was less efficient. Most observers were not aware that the distractor singleton appeared more often in one location than in all other locations. Wang and Theeuwes (2018) interpreted these findings as evidence that statistical regularities unknown to the observer can bias attention such that within the attentional priority map, the location that is likely to contain a distractor singleton is suppressed relative to all other locations. 
Along similar lines, Ferrante et al. (2017) argued that statistical learning can induce plasticity with the spatial priority map such that distractors in particular locations compete less for attention than distractors in other locations.

Several other studies demonstrated that statistical regularities can bias selection. For example, the efficiency of searching for a target can be improved when the target consistently appears in specific locations in previously seen displays relative to random locations (Chun \& Jiang, 1999). Geng and Behrmann (2005) showed that targets presented in high-probability locations are detected faster than those in low-probability locations (see also Jiang, Swallow, Rosenbaum, \& Herzig, 2013). In these studies, in which the target is likely to appear in one particular location, finding benefits in target detection may not be surprising as it is well known that observers can endogenously direct attention to a location in space (Posner, 1980). Indeed, in a series of classic experiments, Posner and others (1980; Posner, Snyder, \& Davidson, 1980) showed that when advance information regarding the location of target is available (usually by means of a location cue), attention will be systematically deployed to this location resulting in faster responding and higher accuracy for targets appearing in this location (i.e., valid trials) than for targets appearing in other locations (i.e., invalid trials) in the visual field. Thus, in tasks in which the target appears in one location more often than in all other locations, it is possible that benefits in responding to the target arise because of what has been labelled as lingering biases of selection history or alternatively because observers implicitly or explicitly direct in a top-down way more attention to the location that is likely to contain a target.

Unlike these previous studies, in Wang and Theeuwes (2018) it was not the target but the distractor that appeared more often in one location than in all other locations. Wang and Theeuwes (2018) claimed that their effects (reduced capture by the distractor singleton and less efficient selection of the target) were due to lingering selection biases, but it is equally likely that observers strategically disregarded this location as it was likely to contain a distractor singleton. Indeed, several previous studies have shown that similar to cuing a high likely target location, cueing a high likely distractor location may also result in a strategic bias of attention such that distractor interference is reduced (Moher \& Egeth, 2012; Munneke, Van der Stigchel, \& Theeuwes, 2008). For example, Munneke et al. (2008) showed that in a display consisting of four letters observers were faster to respond to a target when they were cued by a central arrow to ignore one of the four locations relative to a neutral condition in which no location was cued (see Moher \& Egeth, 2012 for a replication). Others have reported similar findings showing that cueing observers to ignore or inhibit non-target locations can speed up search (e.g., Ruff \& Driver, 2006; Serences, Yantis, Culberson, \& Awh, 2004; Van der Stigchel, Heslenfeld, \& Theeuwes, 2006). Even though previous studies demonstrated that cueing the location of an upcoming distractor reduces its interference with search, it should be noted that distractors employed in these previous studies were never singletons that stood out against the background. It is possible that top-down active inhibition is only effective in displays in which the distractor does not have a largely salient "attend-to-me" signal.

The question is then whether the effects observed by Wang and Theeuwes (2018) are due to lingering biases of selection history or alternatively can be explained by actively suppressing the location that is more likely to contain the distractor singleton. In the current study, we first replicated the basic findings of Wang and Theeuwes (2018) in which the distractor singleton was presented much more often $(65 \%$ of the distractor trials) in one location than in all other locations, and then conducted two similar experiments in which the distractor singleton appeared with a $65 \%$ probability at the location that was cued by a central pointer. Note that in all experiments if a distractor singleton was present, the highprobability location (Experiment 1 ) and the location that was highly likely cued (Experiment 2 and Experiment 3 ) was 13 times more likely to contain the distractor $(65 \%)$ than any of other locations in the display ( $5 \%$ ). In the first experiment we expected to replicate Wang and Theeuwes (2018) showing how statistical regularities can bias attention. The two subsequent experiments tested whether similar results can be obtained by means of active top-down cueing. If active suppression (Experiment 2 and Experiment 3) has the same effect as the biases induced by statistical learning (Experiment 1), we expect (1) reduced capture by the distractor singleton when it appears in the cued location and (2) less efficient selection of the target when it appears in the cued location. If, however, actively ignoring the cued location is not effective, we expect the same capture of the distractor singleton regardless whether it appears in the to-be-ignored location or other locations.

\section{Experiment 1}

The first experiment was a replication of the basic finding of Wang and Theeuwes (2018). We basically used the same experimental setup, which involved a variant of the classic additional singleton task (Theeuwes, 1991, 1992) in which observers searched for a shape singleton (a circle between diamonds or a diamond between circles) while ignoring an irrelevant color singleton (either a green circle or a red circle presented among grey circles). As in Wang and Theeuwes, distractor singletons were presented much more often in one location ( $65 \%$ of the distractor trials) than in other locations ( 5 $\%$ of the distractor trials).

\section{Method}

Participants Twenty adults (18 females, mean age: 19.9 years) from Zhejiang Normal University in China participated in the 
experiment for money compensation. They signed the informed consent and reported normal color vision and normal or corrected-to-normal visual acuity.

Apparatus and stimuli Participants were tested in a dimly lit room with their chin on a chinrest located $63 \mathrm{~cm}$ from the 17in. CRT monitor. To check whether participants maintained fixation, an Eyelink® 1000 eye-tracker was used, with a spatial resolution of $0.2^{\circ}$ or better. The sampling rate of the eyetracker was set to $1,000 \mathrm{~Hz}$. The stimulus presentation and response registration were controlled by custom scripts written in Python.

The stimulus display of Experiment 1 consisted of eight discrete stimuli with different shapes (one circle vs. seven unfilled diamond, or vice versa), each containing a vertical or horizontal grey line $\left(0.3^{\circ} \times 1.5^{\circ}\right)$ inside (see Fig. 1A). These stimuli were presented on an imaginary circle with a radius of $4^{\circ}$, centered at the fixation (a white dot with the radius of $\left.0.25^{\circ}\right)$, against a black background $\left(17 \mathrm{~cd} / \mathrm{m}^{2}\right)$. The circle's radius was $1^{\circ}$, the unfilled diamond was subtended $2^{\circ}$ $\times 2^{\circ}$, and each had a red or green outline.

Procedure and design A self-paced drift check was performed at the beginning of each trial. Then, a fixation dot appeared and remained visible throughout the trial. After $500 \mathrm{~ms}$, the search array was presented for 3,000 ms or until response. Participants had to search for one circle (target) among seven diamonds (distractors), or vice versa, and were required to indicate whether the line segment inside the target was vertical or horizontal, by pressing the "up" or "left" key as fast as possible. The inter-trial interval (ITI) was randomly chosen from $500-750 \mathrm{~ms}$.

The target was present on each trial, and it was equally likely to be a circle or a diamond. $66 \%$ of the trials were distractor singleton present trials, in which a uniquely colored distractor was presented having the same shape as the other distractors, but a different color (red or green with an equal probability). All conditions were randomized within each block. The distractor singleton could be shown in one of eight locations from the imaginary ring with $4^{\circ}$ radius. In the distractor singleton absent (no-distractor) condition, the target appeared in each location with equal chance. The distractor singleton appeared on a high proportion of trials in one particular location, implying that on $65 \%$ of the distractor singleton present trials the distractor singleton appeared in the high-probability location and in $35 \%$ of those trials it appeared equally often in all other locations (low-probability location). The high-probability distractor location remained the same for each participant and was counterbalanced across participants. If a distractor singleton was present, the location that was 13 times more likely to appear in one particular location $(65 \%)$ than any of other locations in the display (5\%).
The experiment consisted of six blocks with each containing 120 trials, and 40 practice trials. Participants had to keep fixation through the trial. If the participants' gaze deviated more than $2^{\circ}$ from fixation, if they did not respond, or if they pressed the wrong key, warning messages were shown. Trials with larger gaze deviation were later retested in a random order until all trials were completed successfully.

\section{Results}

Trials on which the response times (RTs) were faster than $200 \mathrm{~ms}$ and were slower than $1,600 \mathrm{~ms}(5.8 \%)$ were excluded from analyses.

Attentional capture Mean RTs and mean error rates in the distractor singleton present condition are presented in the left panel of Fig. 2A. With distractor condition (high-probability location, low-probability location, and no-distractor) as a factor, a repeated measures ANOVA on mean RTs showed a main effect, $F(2,38)=53.76, p<.001$, partial $\eta^{2}=.74$. Subsequent planned comparisons showed that significant attentional capture effects were observed when a distractor singleton was presented in the high-probability location, $t(19)=$ $4.21, p<.001$, and the low-probability location, $t(19)=8.22$, $p<.001$. Importantly, however, there was also a reliable difference between the high- and low-probability location, $t(19)=7.68, p<.001$.

The results on error rates mimicked those for RTs, With distractor condition (high-probability location, lowprobability location, and no-distractor) as a factor, a repeated measures ANOVA showed a main effect, $F(2,38)=26.12, p$ $<.001$, partial $\eta^{2}=.58$. Subsequent planned comparisons showed that significant attentional capture effects were observed when a distractor singleton was presented in the highprobability location, $t(19)=3.07, p=.006$, and the lowprobability location, $t(19)=5.99, p<.001$. Importantly, however, there was also a reliable difference between the highand low-probability location, $t(19)=5.07, p<.001$. Taken together, these results suggest that attentional capture effect was reduced for trials in which the distractor singleton appeared in a high-probability relative to the low-probability location, a straightforward replication of Wang and Theeuwes (2018).

Efficiency of selection To determine whether the efficiency of selecting the target was affected, the RTs and error rates in the no-distractor condition were examined. As shown in the left panel of Fig. 2B, the results showed that participants were slower to respond when the target was presented in the highprobability distractor location compared to the lowprobability distractor location, $t(19)=4.63, p<.001$. There was no effect on error rates, $t(19)=1.14, p=.268$. 


\section{A) Experiment 1}

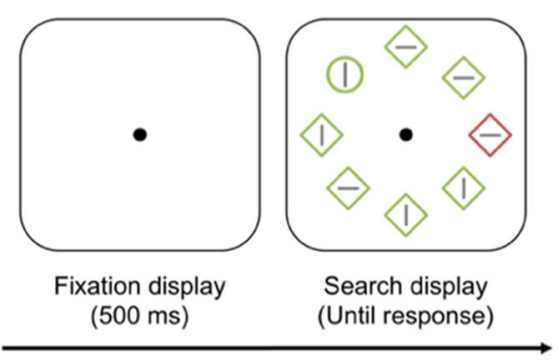

\section{B) Experiment 2}

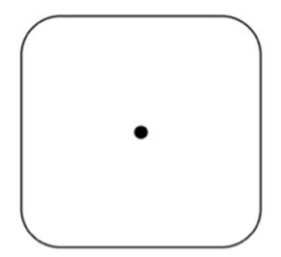

Fixation display (500 ms)

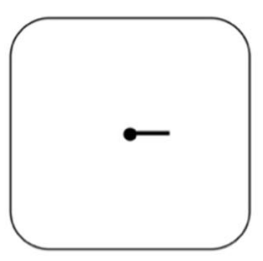

Cueing display (1050 ms)

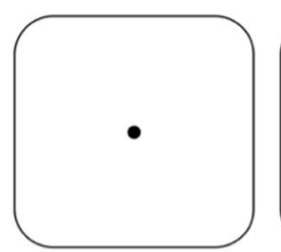

Interval

(450 ms)

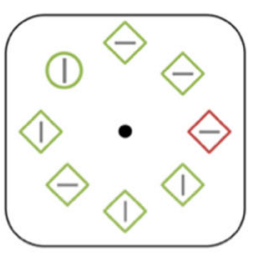

Search display (Until response)

Fig. 1 The sequence of a single trial in Experiment 1 (A) and Experiment 2 (B). Experiment 3 had the same trial sequence as in Experiment 2, except that the cue was presented for $150 \mathrm{~ms}$ only

\section{A) Distractor singleton present}
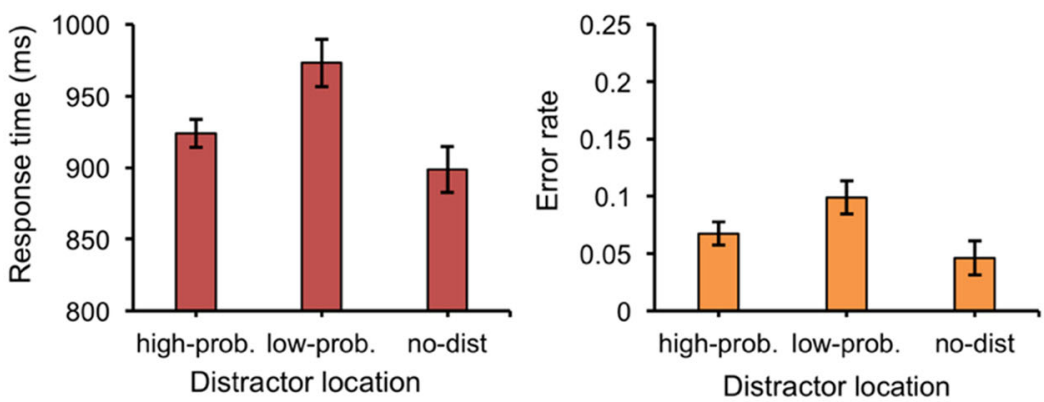

B) Distractor singleton absent (no-distractor condition)
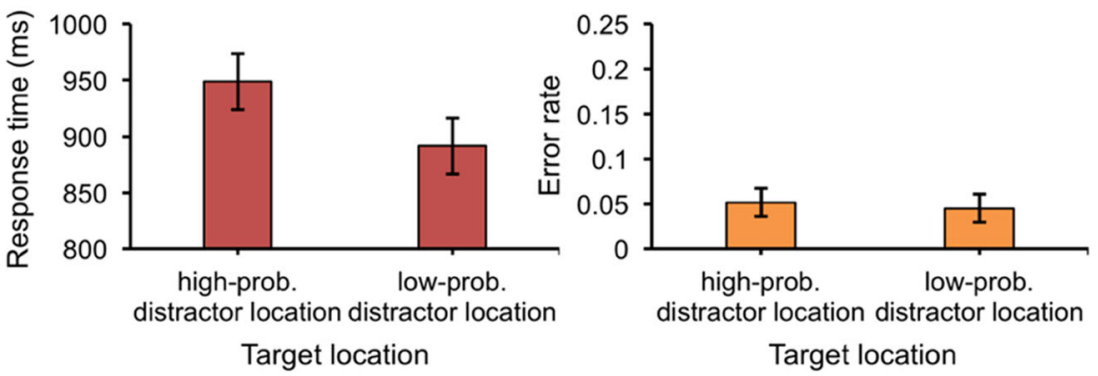

Fig. 2 The main replication results of Wang and Theeuwes in Experiment 1. (A) The mean response times (RTs; left panel) and the mean error rates (right panel) between different distractor conditions. (B) The mean RTs

(left panel) and the mean error rates (right panel) in the no-distractor condition. Error bars denote within-subjects $95 \%$ confidence intervals 
The spatial gradient of the suppression effect To explore the spatial distribution of the suppression effect, the distractor locations were divided into five distances (dist- 0 , dist- 1 , dist2 , dist-3, and dist-4) ${ }^{1}$ from the high-probability distractor location. The mean RTs and mean error rates for these conditions are presented in Fig. 3A. A repeated measures ANOVA on mean RTs with distance as a factor showed a significant main effect, $F(4,76)=13.16, p<.001$, partial $\eta^{2}=.41$. The same effect was found for error rates, $F(4,76)=3.38, p=.038$, partial $\eta^{2}=.15$. Moreover, we fitted the data with linear functions and used their slope to determine whether the mean RTs and mean error rates changed with distance. The slopes (20.31 ms and $1.2 \%$ per display element) were significantly larger than zero, $t(19)=7.21, p<.001$ and $t(19)=3.11, p=$ .006 , respectively, suggesting a spatial gradient (the suppression effect grew when the distractor singleton was presented farther away from the high-probability distractor location).

In the no-distractor condition, there was also a significant main effect on mean RTs for distance, $F(4,76)=8.17, p<$ .001 , partial $\eta^{2}=.3$. The mean RTs and mean error rates for these conditions are presented in Fig. 3B. The gradient effect was now reversed: With increased distance, the selection of the target became progressively more efficient, signified by a slope of $-11.51 \mathrm{~ms}$ per display element that differed from zero, $t(19)=3.4, p=.003$. However, as is clear from Fig. 3B, this effect is largely driven by the drop in RT from distance zero (dist-0) to distance 1 (dist-1). Regardless of whether suppression takes the form of a gradient, it is important to note that suppression also occurred in the condition in which there was no distractor.

Short-term location-based priming? One concern is that the current effects are not due to statistical learning but instead are completely driven by trial-by-trial location-based priming. Because the distractor appears much more often in one location (the high-probability location) than in all other locations it is feasible that it is not statistical learning as such but lingering suppression effects from the previous trial that drives the effect. To test this, we analyzed trials in which the distractor singleton was repeated in the high-probability location versus trials in which it was presented in the preceding trial in another (low-probability) location. The mean RTs for repeated trials (from high-probability distractor location to high-probability distractor location) was $923 \mathrm{~ms}$ and the mean RTs for nonrepeated trials (from low-probability distractor location to high-probability distractor location) was $924 \mathrm{~ms}$. Clearly the difference was negligible, and statistically not reliable, $t(19)=$

\footnotetext{
${ }^{1}$ The dist- 0 location refers to the high-probability distractor location; the dist-1 location means that the distractor was presented next to the high-probability distractor location $\left(45^{\circ}\right.$ polar angle); the dist- 2 location refers to $90^{\circ}$ from the high-probability distractor location; the dist-3 location refers to $135^{\circ}$ from the high-probability distractor location; and the dist- 4 location refers to $180^{\circ}$ from the high-probability distractor location.
}

$0.71, p=.484$, with a Bayes Factor (BF) of 3.43. Moreover, there was also a spatial gradient of the suppression effect on RTs and error rates when analyzing only trials in which the location was not repeated, $F(4,76)=12.45, p<.001$, partial $\eta^{2}$ $=.4$ and $F(4,76)=3.3, p=.015$, partial $\eta^{2}=.15$, respectively. The slopes (19.95 ms and $1.2 \%$ per display element) were significantly larger than zero, $t(19)=6.9, p<.001$ and $t(19)=$ $3.17, p=.005$, respectively. On the basis of these results, we conclude that short-term location-based priming of the distractor location cannot explain the suppression effects that we observed here (see also Wang \& Theeuwes, 2018; for similar analyses and conclusions) and is unlikely to be the factor driving the effect.

\section{Discussion}

Experiment 1 replicates our previous findings in all details showing (1) reduced capture by the distractor singleton when it appears in a high-probability location relative to lowprobability locations and (2) a reduced efficiency of selecting the target when it happened to be presented in the highprobability location relative to all other locations. We found a spatial gradient of suppression both for when a distractor was presented in that location (more capture the further away from this location) and also in the no-distractor condition when the target happened to be presented here (faster RTs the further away from this location).

These findings are interpreted as evidence that statistical regularities bias attention such that a location that is more likely to contain a distractor is suppressed relative to all other locations. The finding of a spatial gradient of suppression both for the distractor (the closer to the suppressed location, the less capture was observed) and the target (the closer to the suppressed location, the less efficient target selection was) around this location provides converging evidence that statistical learning can induce plasticity within the spatial priority map (see also Ferrante et al., 2017).

\section{Experiment 2}

Experiment 2 had a similar design as Experiment 1 except that now the location that was likely to contain a distractor singleton was cued on every trial. Similar to Experiment 1, if a distractor singleton was present, the cued location was 13 times more likely to contain a distractor singleton $(65 \%)$ than any of other locations in the display ( $5 \%)$. There is every reason for participants to use this cue to improve their performance. Any effect of the cue on performance would imply that observers actively use the cue. With this manipulation, we are ensured that if an effect of the cue on performance would be found, the effect would be truly top-down in nature. As outlined in a recent review by Theeuwes (2018), in order to 
A) Singleton distractor present
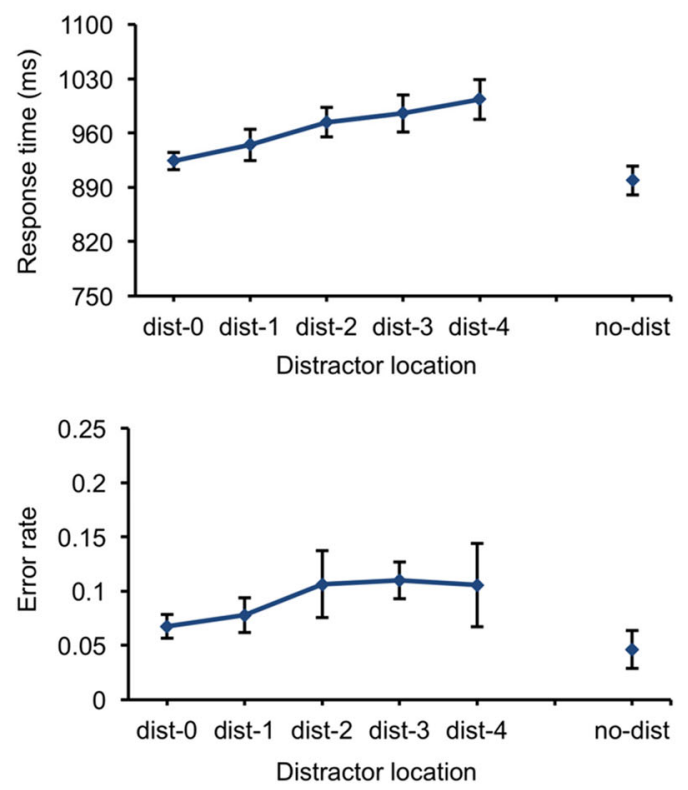

Fig. 3 The spatial distribution of attentional capture effect by the means of response times (RTs) (upper panel) and error rates (lower panel) in Experiment 1 (A). The spatial distribution of selection efficient by the means of RTs (upper panel) and error rates (lower panel) in Experiment 1

establish top-down effects on selection, it is important that participants set up a specific top-down goal on each and every trial. Only then one can infer true top-down, volitional effects on selection (see also Belopolsky, Schreij, \& Theeuwes, 2010; Theeuwes, 2013). A good example of volitional top-down control on visual selection is achieved in the classic Posner endogenous cueing task in which participants are cued on each and every trial by a central arrow (or word) to direct their attention at will to a specific location in space (Posner, 1980; Theeuwes \& Van der Burg, 2007).

In Experiment 2 we employed a stimulus-onset asymmetry (SOA) of $1,500 \mathrm{~ms}$, which is identical to that used in Munneke et al.'s (2008) distractor cueing study (see also Moher \& Egeth, 2012, and Chao, 2010 who also used 1,500 ms). Such a long SOA should give ample opportunity to establish a top-down set for inhibiting the location of the impending distractor singleton.

\section{Method}

Twenty adults (17 females, mean age: 19.8 years) from Zhejiang Normal University in China participated in the current experiment for money compensation. They reported normal color vision and normal or corrected-to-normal visual acuity. The stimuli, procedure, and experimental design were the same as Experiment 1, except that the distractor singleton was equally likely to appear in any of the locations. We presented a cue (a pointer) indicating the location that was likely to contain the distractor singleton. Figure 1B gives an example of the trial
B) Singleton distractor absent
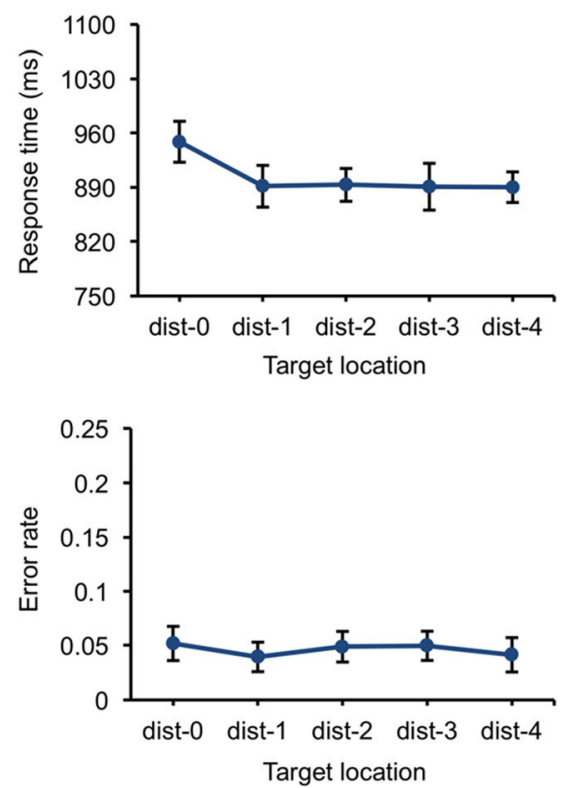

(B). Here Dist-0 refers to the high-probability distractor location, Dist-1 refers to the low-probability distractor location with one unit $\left(45^{\circ}\right.$ polar angle) away from the high-probability distractor location, and so on. Error bars denote within-subjects $95 \%$ confidence intervals

sequence. The cue was presented for $1,050 \mathrm{~ms}$ followed by an inter-stimulus interval (ISI) of $450 \mathrm{~ms}$ (i.e., an SOA of 1,500 $\mathrm{ms})$. In the distractor singleton present condition, the cued location was 13 times more likely to contain a distractor singleton ( $65 \%$; validly cued location) than any of other locations in the display ( $5 \%$; invalidly cued location). In the no-distractor condition, each location was indicated by the cue with equal chance. Participants were explicitly told that the cue pointed to the location that was likely to contain a distractor singleton, and that they should make use of this information to improve performance.

\section{Results}

Trials on which the response times (RTs) were faster than $200 \mathrm{~ms}$ and were slower than $1,600 \mathrm{~ms}$ ( $8.9 \%$ in total) were excluded from analyses.

Attentional capture Mean RTs and mean error rates are presented in Fig. 4A. With cue condition (valid cue, invalid cue, and no-distractor) as a factor, mean RTs were entered into a repeated measures ANOVA and showed a main effect, $F(2$, $38)=71.46, p<.001$, partial $\eta^{2}=.79$. Subsequent comparisons showed that there were significant attentional capture effects for a distractor singleton presented in the validly cued location, $t(19)=8.74, p<.001$, and for a distractor singleton presented in the invalidly cued location, $t(19)=10.19, p<$ .001. Importantly, however, there was no difference between the validly and invalidly cued conditions, $t(19)=0.18, p=$ .863 , with a $\mathrm{BF}$ of 3.1 , suggesting that a cue indicating the 
A) Distractor singleton present
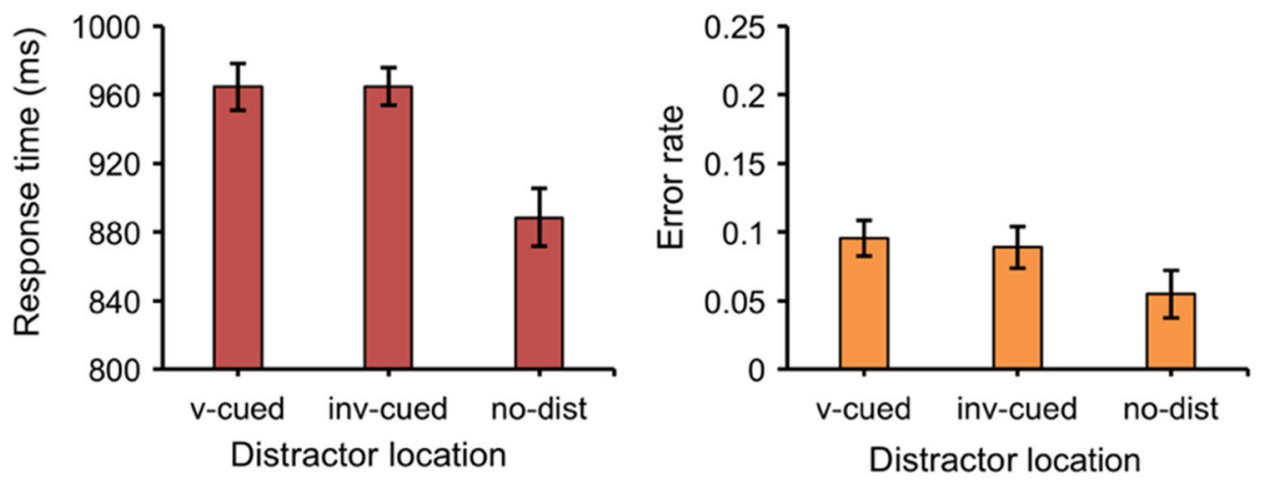

B) Distractor singleton absent (no-distractor condition)

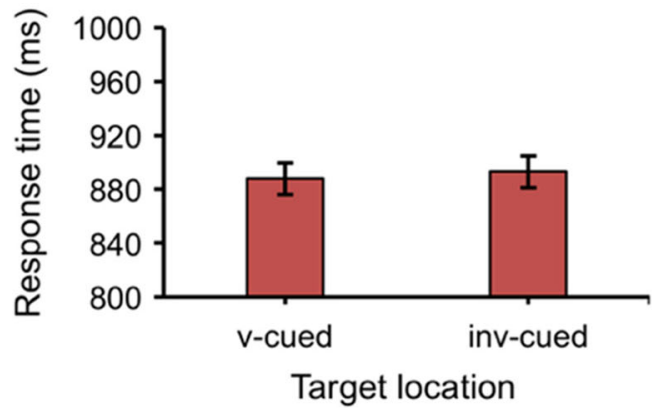

Fig. 4 The comparison on response times (RTs) (left panel) and error rates (right panel) between valid and invalid cued location in Experiment 2. The distractor singleton present and no-distractor

location of the upcoming distractor singleton does not eliminate attentional capture.

Error rates mimicked those of RTs. A repeated measures ANOVA on mean error rates with cue condition (valid cue, invalid cue, and no-distractor) as a factor showed a main effect, $F(2,38)=12.57, p<.001$, partial $\eta^{2}=.4$. Subsequent comparisons showed that participants made more errors in validly cued and invalidly cued conditions relative to the no-distractor condition, $t(19)=4.63, p<.001$ and $t(19)=3.46, p<.001)$, respectively. There was no difference in error rates between the validly and invalidly cued conditions, $t(19)=0.91, p=.373$, with a BF of 4.04. The spatial gradient analysis of cueing effect in the present and following experiments was in Appendix.

Efficiency of selection Target selection efficiency was also not affected by the cue: There was no difference in RTs and error rates in the distractor singleton absent condition, $t(19)=0.5, p$ $=.622$, with a BF of 3.84, and $t(19)=0.49, p=.631$, with a BF of 3.86, respectively (see Fig. 4B).

\section{Discussion}

Experiment 2 shows that cueing the likely location of the distractor singleton on a trial-by-trial basis had no effect on

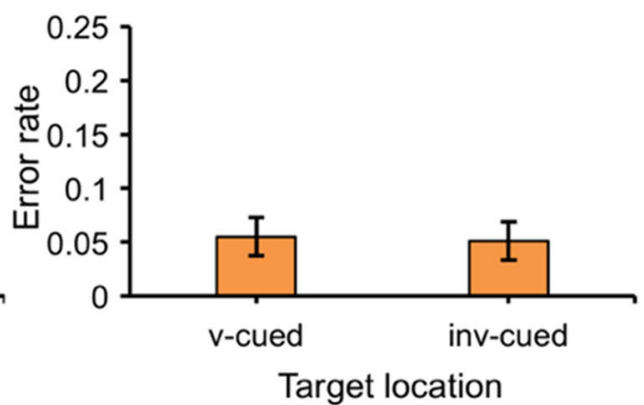

conditions are present in figures $(\mathbf{A})$ and $(\mathbf{B})$, respectively. Error bars denote within-subjects $95 \%$ confidence intervals

capture nor on the efficiency of target selection, suggesting that people could not actively inhibit the cued location. Even though we used the same long SOA as used in previous studies (Chao, 2010; Moher \& Egeth, 2012; Munneke et al., 2008) there was no evidence for any suppression of that location. Note that the location that was cued 13 times more often to contain a distractor singleton than any of the other locations. Even though observers knew that the cue pointed to the likely distractor location, they were unable to use this information to actively suppress it. This is very much unlike what we saw in Experiment 1 in which a lingering selection history biased attention such that the location that was more likely to contain a distractor singleton was suppressed relative to all other locations.

\section{Experiment 3}

One concern that we had with Experiment 2 is that the SOA of 1,500 ms may have been too long to obtain active suppression. Even though long SOAs were also used in previous studies (e.g., Munneke et al., 2008) it is possible that with this long SOA active suppression cannot be maintained in the cued location. To test this, we re-ran Experiment 2 but now with a shorter SOA of $600 \mathrm{~ms}$. 


\section{Method}

Twenty adults (17 females, mean age: 19.6 years) from Zhejiang Normal University in China participated in the current experiment for money compensation. They reported normal color vision and normal or corrected-to-normal visual acuity. The stimuli, procedure, and experimental design were identical to Experiment 2, except that the cue indicating the likely distractor location was presented for $150 \mathrm{~ms}$ followed by an ISI of $450 \mathrm{~ms}$, resulting in an SOA of $600 \mathrm{~ms}$.

\section{Results}

Trials on which the response times (RTs) were faster than $200 \mathrm{~ms}$ and were slower than $1,600 \mathrm{~ms}(6.1 \%$ in total $)$ were excluded from the analyses.

Attentional capture Mean RTs and mean error rates are presented in Fig. 5A. With cue condition (valid cue, invalid cue, and no-distractor) as a factor, mean RTs were entered into a repeated measures ANOVA and showed a main effect, $F(2$, $38)=85.84, p<.001$, partial $\eta^{2}=.82$. Subsequent comparisons showed that there were significant attentional capture effects for a distractor singleton presented in the validly cued location, $t(19)=10.2, p<.001$, and for a distractor singleton presented in the invalidly cued location, $t(19)=9.94, p<.001$. Importantly, however, there was again no difference between validly and invalidly cued conditions, $t(19)=0.26, p=.798$, with a BF of 3.24, indicating that also for the shorter SOA there was no evidence for active suppression of the location of the upcoming distractor singleton.

Error rates mimicked those on RTs. A repeated measures ANOVA on mean error rates with cue condition (valid cue, invalid cue, and no-distractor) as a factor showed a main effect, $F(2,38)=17.67, p<.001$, partial $\eta 2=.48$. Subsequent comparisons showed that participants made more errors in validly cued and invalidly cued conditions relative to the nodistractor condition, $t(19)=4.16, p<.001$ and $t(19)=5.26, p$ $<.001$, respectively. There was no difference in error rates between the validly and invalidly cued conditions, $t(19)=$ $0.62, p=.541$, with a BF of 3.77 .

Efficiency of selection The no-distractor condition showed that when the target happened to be presented in the cued location participants were faster than when the target appeared in noncued locations $t(19)=2.13, p=.047$ (see Fig. 5B). So even though participants knew that the distractor singleton was very likely to be presented at the cued location, the results show

\section{A) Distractor singleton present}
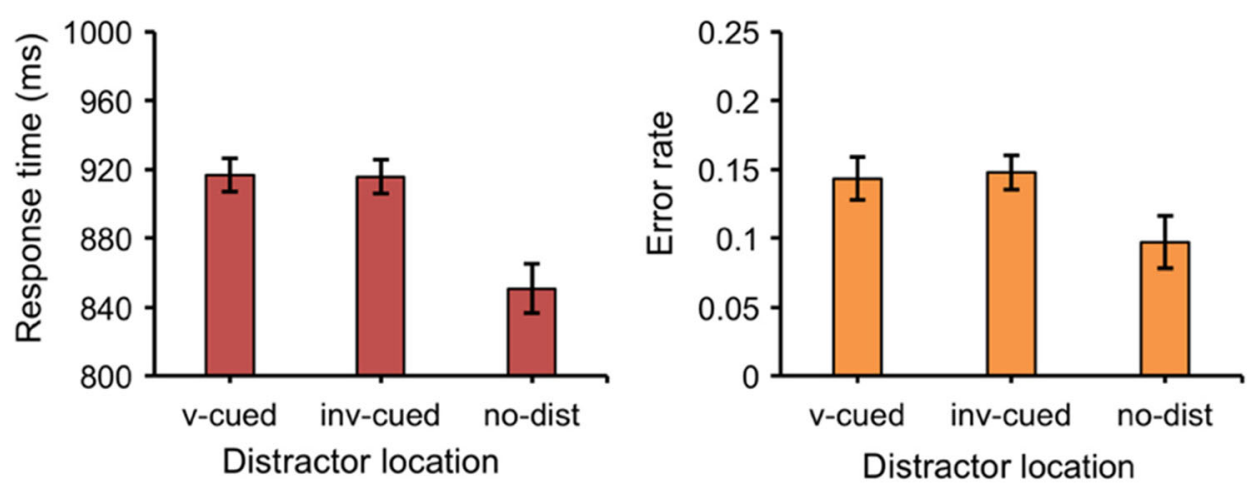

B) Distractor singleton absent (no-distractor condition)

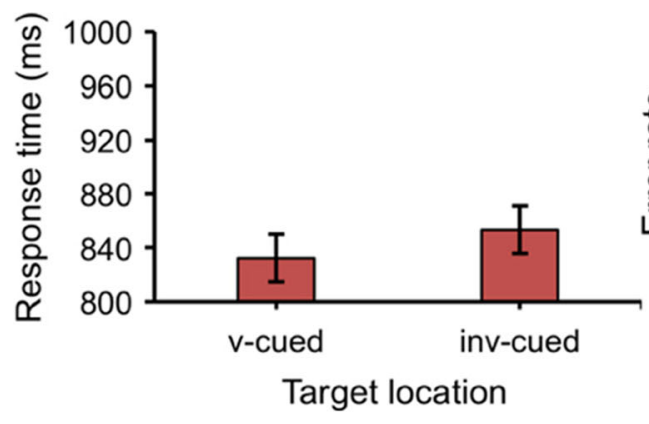

Fig. 5 Comparison of response times (RTs) (left panel) and error rates (right panel) between valid and invalid cued location in Experiment 3. The distractor singleton present and no-distractor conditions are present

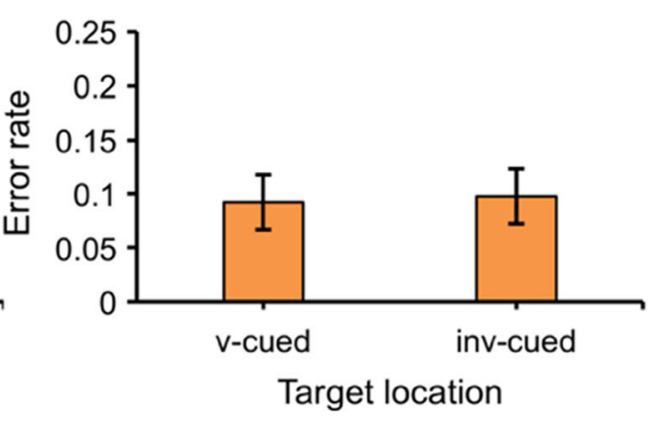

in figures (A) and (B), respectively. Error bars denote within-subjects 95 $\%$ confidence intervals 
that they were nevertheless faster when on those few trials the target was presented there. There was no effect on error rates, $t(19)=0.41, p=.684$, with a BF of 3.99 .

\section{Discussion}

The current experiment again showed that cueing the likely location of the distractor singleton on a trial by trial basis had no effect on capture nor on the efficiency of selection. If anything, the current experiment shows that the attempt of participants to actively inhibit the cued location only resulted in attentional benefits: If the target happened to be presented at the cued location, participants were slightly faster in selecting the target $(12 \mathrm{~ms})$ than when it appeared in a different location. This finding is important as it suggests that participants did not simply ignore the cue but instead tried to use the cue to improve performance within a short period $(600 \mathrm{~ms})$.

\section{General discussion}

The current study shows that introducing statistical regularities can bias attention such that the location that is likely to contain a distractor singleton is suppressed relative to all other locations (as in Wang \& Theeuwes, 2018). Also, we show a spatial gradient of attentional suppression for target and distractor that extends with the distance from the suppressed location. However, cueing the location of the distractor singleton does not induce the same suppression effect: Neither the amount of capture nor the efficiency of selection was affected by the cue. This was the case for both a relatively long cue-distractor SOA of 1,500 ms (Experiment 2) and a short SOA of $600 \mathrm{~ms}$ (Experiment 3 ). We argue that the effects observed in Experiment 1 and in Wang and Theeuwes (2018) are the result of lingering biases of selection history unrelated to top-down and bottom-up control mechanism (Awh et al., 2012; Theeuwes, 2018). This type of statistical learning, rather than active inhibition, changes the weights within the attentional priority map (see also Ferrante et al., 2017) that controls the allocation of attention within the visual field.

Recently, Gaspelin and Luck $(2017,2018)$ suggested a topdown suppression account referred to as the "signal suppression" hypothesis stating that through spatial inhibition it is possible to avoid capture by salient signals (see also Sawaki \& Luck, 2010). The underlying idea is that even though all salient stimuli generate a strong bottom-up signal, capture can be avoided by suppressing the activity of the salient stimulus in a top-down way. Evidence for the signal suppression hypothesis comes from two important findings. On the one hand, the active suppression process can be indexed by the distractor positivity (Pd), a component of the event-related potential (ERP) waveform (Hickey, Di Lollo, \& McDonald, 2008; Sawaki \& Luck, 2010). Several studies have shown that there is no attentional capture by salient stimuli, when a Pd component is observed in the ERP signal (e.g., Burra \& Kerzel, 2014; Eimer \& Kiss, 2008; Sawaki \& Luck, 2010). On the other hand, behavioral evidence from what is known as the probe dot task originally pioneered by Kim and Cave (1995) showed that participants were less likely to report a probe presented in the location of the salient distractor than at all other locations (Gaspelin, Leonard, \& Luck, 2015). This suggests that the location of the salient distractor is suppressed below baseline processing. Also, eye-tracking data showed similar results: Participants made fewer eye movements to the location of the salient distractor than to non-salient locations, again suggesting some form of suppression (Gaspelin, Leonard, \& Luck, 2017).

The notion of distractor suppression advocated by Gaspelin and Luck (2018) is very similar to our views regarding suppression except that we do not claim that this effect is topdown in origin. Gaspelin and Luck argue that "A salient-butirrelevant singleton can be actively suppressed when topdown guidance is deployed" (Gaspelin et al., 2015, p. 9). This is exactly what we tried to do here: By providing a cue that indicated the likely location of the distractor we induced this top-down guidance that should according to Gaspelin and Luck (2017) have resulted in active suppression. However, there was no evidence for this; instead statistical learning (i.e., selection history) did generate the similar type of suppression to that described by Gaspelin and Luck (2017). Note, however, that unlike Gaspelin and Luck (2017) in our studies suppression of the high-probability location was never perfect, as a small but reliable capture effect remained present in all our studies (Wang \&Theeuwes, 2018).

In the current study, cueing the distractor location on a trialby-trial basis was not effective in inhibition distractor processing. If anything, Experiment 3 showed that at a short SOA instead of suppressing the distractor location, attention was allocated to that location such that if the target happened to be presented there, participants were faster to respond than when it appeared in non-cued locations. This is very similar to findings of Moher and Egeth (2012) in which participants were cued to inhibit particular distractor features (e.g., say the color red). They showed that initially the location containing the to-be-ignored feature was attended before inhibition could be applied. That is, instead of ignoring this feature, participants could not prevent attending this feature. Only when the premask containing the to-be-ignored feature were presented at least $800 \mathrm{~ms}$ before the search display participants were able to ignore it. Even though on the face of it this study may appear to be about feature suppression, Moher and Egeth (2012) indicated that this inhibition that occurred so late in time is probably purely location-based, and in that respect not different from Munneke et al. (2008). Moher and Egeth (2012) dubbed this the "search and destroy" strategy indicating that one first has to attend to a location before one is able to 
inhibit it. Note that this mechanism is completely different from Gaspelin and Luck's $(2017,2018)$ "signal suppression" hypothesis because the latter assumes suppression of feature without attention first being deployed to that location. Regardless of whether it is a "search and destroy" strategy (Moher \& Egeth, 2012) or a top-down "signal suppression" mechanism (Gaspelin \& Luck, 2017), the current findings indicate that top-down cuing on a trial-by-trial basis does not result in inhibition of the distractor location. Only statistical learning leads to suppression of the distractor location.

One may question whether participants were motivated enough to use the cue. The absence of an effect of the cue may indicate that participants actively tried to use the cue to inhibit the location but this attempt was not successful (this is what we conclude here), or, alternatively, that participants did not bother to use the cue at all. Even though this latter explanation is conceivable, it is not likely for two reasons. First, we do see a small (but reliable) effect in Experiment 3, indicating that participants did not simply ignore the cue but actively tried to use it on a trial by trial basis. Obviously, this attempt did not lead to inhibition of the cued location (an activation at best) but it does argue against the idea that participants simply ignored the cue. Second, several other cueing studies from different labs that have used an SOA of 1,500 ms (Chao, 2010; Moher \& Egeth, 2012; Munneke et al., 2008) have shown robust suppression effects suggesting that with this setup and this SOA, in other paradigms that do not use salient distractors, top-down inhibition was successful. Therefore, it is unlikely that in previous studies using the same setup, participants actively used the cue while in our experiments, for some reason, they decided to ignore the cue.

One question that remains unanswered is why a "search and destroy" mechanism as described by Moher and Egeth (2012) does not work in our paradigm. We do see something comparable to their "search" mechanism, i.e., at the short SOA of $600 \mathrm{~ms}$, the to-be-ignored location was attended. Yet, there is no evidence of subsequent inhibition at the longer SOA of $1,500 \mathrm{~ms}$, even though this SOA is much longer than the minimum of $800 \mathrm{~ms}$ SOA suggested by Moher and Egeth (2012) and identical to the SOAs used by Munneke et al. (2008) and Chao (2010). One explanation is that previous studies that have shown active inhibition on the basis of trial-by-trial cueing (e.g., Munneke et al., 2008; Moher \& Egth, 2012) have used non-salient distractors. It is feasible that the "search and destroy" strategy works but only in displays in which the distractor does not have a largely salient "attend-to-me" signal. In addition, these previous studies have used fewer display elements (typically four instead of eight as in the current study), which makes it possible that instead of actively inhibiting the cued location, participants actively enhance all locations to which the cued does not point. This strategy is basically impossible with eight display elements (see Munneke et al., 2008 for a similar argument).
We conclude that the irrelevant salient signals that are known to capture attention in singleton search can be suppressed by implicit statistical learning (i.e., lingering biased selection history). Our findings suggest that in the current setup this cannot be done by active top-down inhibition. Through statistical learning, weights within the attentional priority map are changed such that the location that is likely to contain a salient distractor is suppressed relative to all other locations. Changing weights within the priority map cannot be accomplished by active, top-down suppression.

Author notes The authors thank three anonymous reviewers for their helpful suggestions. This research was supported by a European Research Council (ERC) advanced grant [ERC2012-AdG-323413] to JT and a China Scholarship Council (CSC) scholarship [201508330313] to BW.

\section{Appendix: Evaluating the spatial distribution of cueing effect in Experiments 2 and 3.}

\section{Experiment 2}

To check the spatial distribution of the cueing effect, the distractor locations were divided into five distances (dist- 0 , dist-1, dist-2, dist-3, and dist-4) from the cued location. A repeated measures ANOVA on mean RTs with distance as a factor showed null effect, $F(4,76)=1.21, p=.312$, partial $\eta^{2}=.06$, with a BF of 5.36. The effect was significant for error rates, $F(4$, 76) $=3.1, p=.02$, partial $\eta^{2}=.14$. However, importantly, we fitted the data with linear functions and used their slope to determine whether the mean error rates changed with distance. The slopes $(0.6 \%$ per display element) were not significantly larger than zero, $t(19)=1.94, p=.068$, with a BF of 1.1. Also, in the no-distractor condition, there were no significant main effects on mean RTs and error rates for distance, $F(4,76)=0.78, p=.54$, partial $\eta^{2}=.04$, with a BF of 9.37 , and $F(4,76)=0.49, p=.745$, partial $\eta^{2}=.03$, with a BF of 13.8 , respectively.

\section{Experiment 3}

Same as the analysis above, a repeated measures ANOVA with distance as a factor showed a main effect for RTs, $F(4,76)=$ $2.91, p=.027$, partial $\eta^{2}=.13$; but not for error rates, $F(4,76)=$ $2.4, p=.058$, partial $\eta^{2}=.11$, with a BF of 1.14 . However, importantly, we fitted the data with linear functions and used their slope to determine whether the mean RTs changed with distance. The slopes (1.8 ms per display element) were not significantly larger than zero, $t(19)=0.93, p=.364$, with a BF of 2.93. Also in the no-distractor condition, there were no significant main effects on mean RTs and error rates for distance, $F(4,76)=$ $1.6, p=.183$, partial $\eta^{2}=.08$, with a BF of 3.22 , and $F(4,76)$ $=0.11, p=.979$, partial $\eta^{2}=.01$, with a BF of 22.67 , respectively. 


\section{References}

Anderson, B. A. (2016). The attention habit: How reward learning shapes attentional selection. Annals of the New York Academy of Sciences, 1369(1), 24-39. https://doi.org/10.1111/nyas.12957

Awh, E., Belopolsky, A. V., \& Theeuwes, J. (2012). Top-down versus bottom-up attentional control: A failed theoretical dichotomy. Trends in Cognitive Sciences, 16(8), 437-443. https://doi.org/10. 1016/j.tics.2012.06.010

Belopolsky, A. V., Schreij, D., \& Theeuwes, J. (2010). What is top-down about contingent capture? Attention, Perception, \& Psychophysics, 72(2), 326-341. https://doi.org/10.3758/APP.72.2.326

Burra, N., \& Kerzel, D. (2014). The distractor positivity (Pd) signals lowering of attentional priority: Evidence from event-related potentials and individual differences. Psychophysiology, 51(7), 685-696. https://doi.org/10.1111/psyp.12215

Chao, H.-F. (2010). Top-down attentional control for distractor locations: The benefit of precuing distractor locations on target localization and discrimination. Journal of Experimental Psychology: Human Perception and Performance, 36(2), 303.

Chun, M. M., \& Jiang, Y. (1999). Top-down attentional guidance based on implicit learning of visual covariation. Psychological Science, 10(4), 360-365. https://doi.org/10.1111/1467-9280.00168

Eimer, M., \& Kiss, M. (2008). Involuntary attentional capture is determined by task set: Evidence from event-related brain potentials. Journal of Cognitive Neuroscience, 20(8), 1423-1433. https://doi. org/10.1162/jocn.2008.20099

Ferrante, O., Patacca, A., Di Caro, V., Della Libera, C., Santandrea, E., \& Chelazzi, L. (2017). Altering spatial priority maps via statistical learning of target selection and distractor filtering. Cortex. https:// doi.org/10.1016/j.cortex.2017.09.027

Gaspelin, N., Leonard, C. J., \& Luck, S. J. (2015). Direct evidence for active suppression of salient-but-irrelevant sensory inputs. Psychological Science, 26(11), 1740-1750. https://doi.org/10. $1177 / 0956797615597913$

Gaspelin, N., Leonard, C. J., \& Luck, S. J. (2017). Suppression of overt attentional capture by salient-but-irrelevant color singletons. Attention, Perception, \& Psychophysics, 79(1), 45-62. https://doi. org/10.3758/s13414-016-1209-1

Gaspelin, N., \& Luck, S. J. (2017). Distinguishing among potential mechanisms of singleton suppression. Journal of Experimental Psychology. Human Perception and Performance. https://doi.org/ 10.1037/xhp0000484

Gaspelin, N., \& Luck, S. J. (2018). The role of inhibition in avoiding distraction by salient stimuli. Trends in Cognitive Sciences, 22(1), 79-92. https://doi.org/10.1016/j.tics.2017.11.001

Geng, J. J., \& Behrmann, M. (2005). Spatial probability as an attentional cue in visual search. Perception \& Psychophysics, 67(7), 12521268. https://doi.org/10.3758/BF03193557

Hickey, C., Di Lollo, V., \& McDonald, J. J. (2008). Electrophysiological indices of target and distractor processing in visual search. Journal of Cognitive Neuroscience, 21(4), 760-775. https://doi.org/10.1162/ jocn.2009.21039

Jiang, Y. V. (2017). Habitual versus goal-driven attention. Cortex. https:// doi.org/10.1016/j.cortex.2017.06.018
Jiang, Y. V., Swallow, K. M., Rosenbaum, G. M., \& Herzig, C. (2013). Rapid acquisition but slow extinction of an attentional bias in space. Journal of Experimental Psychology: Human Perception and Performance, 39(1), 87-99. https://doi.org/10.1037/a0027611

Kim, M.-S., \& Cave, K. R. (1995). Spatial attention in visual search for features and feature conjunctions. Psychological Science, 6(6), 376380. https://doi.org/10.1111/j.1467-9280.1995.tb00529.x

Moher, J., \& Egeth, H. E. (2012). The ignoring paradox: Cueing distractor features leads first to selection, then to inhibition of tobe-ignored items. Attention, Perception, \& Psychophysics, 74(8), 1590-1605. https://doi.org/10.3758/s13414-012-0358-0

Munneke, J., Van der Stigchel, S., \& Theeuwes, J. (2008). Cueing the location of a distractor: An inhibitory mechanism of spatial attention? Acta Psychologica, 129(1), 101-107. https://doi.org/10.1016/ j.actpsy.2008.05.004

Posner, M. I. (1980). Orienting of attention. The Quarterly Journal of Experimental Psychology, 32(1), 3-25.

Posner, M. I., Snyder, C. R., \& Davidson, B. J. (1980). Attention and the detection of signals. Journal of Experimental Psychology, 109(2), $160-174$.

Ruff, C. C., \& Driver, J. (2006). Attentional preparation for a lateralized visual distractor: Behavioral and fMRI evidence. Journal of Cognitive Neuroscience, 18(4), 522-538. https://doi.org/10.1162/ jocn.2006.18.4.522

Sawaki, R., \& Luck, S. J. (2010). Capture versus suppression of attention by salient singletons: Electrophysiological evidence for an automatic attend-to-me signal. Attention, Perception, \& Psychophysics, 72(6), 1455-1470. https://doi.org/10.3758/APP.72.6.1455

Serences, J. T., Yantis, S., Culberson, A., \& Awh, E. (2004). Preparatory activity in visual cortex indexes distractor suppression during covert spatial orienting. Journal of Neurophysiology, 92(6), 3538-3545. https://doi.org/10.1152/jn.00435.2004

Theeuwes, J. (1991). Cross-dimensional perceptual selectivity. Perception \& Psychophysics, 50(2), 184-193. https://doi.org/10. 3758/BF03212219

Theeuwes, J. (1992). Perceptual selectivity for color and form. Perception \& Psychophysics, 51(6), 599-606. https://doi.org/10.3758/ BF03211656

Theeuwes, J. (2013). Feature-based attention: It is all bottom-up priming. Philosophical Transactions of the Royal Society of London B: Biological Sciences, 368(1628), 20130055. https://doi.org/10. 1098/rstb.2013.0055

Theeuwes, J. (2018). Visual selection: usually fast and automatic; seldom slow and volitional. Journal of Cognition. https://doi.org/10.5334/ joc. 13

Theeuwes, J., \& Van der Burg, E. (2007). The role of spatial and nonspatial information in visual selection. Journal of Experimental Psychology: Human Perception and Performance, 33(6), 1335.

Van der Stigchel, S., Heslenfeld, D. J., \& Theeuwes, J. (2006). An ERP study of preparatory and inhibitory mechanisms in a cued saccade task. Brain Research, 1105(1), 32-45. https://doi.org/10.1016/j. brainres.2006.02.089

Wang, B., \& Theeuwes, J. (2018). Statistical regularities modulate attentional capture. Journal of Experimental Psychology: Human Perception and Performance, 44(1), 13-17. https://doi.org/10. 1037/xhp0000472 